\title{
Dynamic Behaviours of a Single Soft Rock-Socketed Shaft Subjected to Axial Cyclic Loading
}

\author{
Ben-jiao Zhang, ${ }^{1}$ Bin Huang, ${ }^{1,2}$ Can Mei, ${ }^{1}$ Xu-dong Fu, ${ }^{1}$ Gang Luo, ${ }^{1}$ and Zhi-jun Yang ${ }^{1}$ \\ ${ }^{1}$ School of Civil and Architectural Engineering, Wuhan University, Wuhan 430072, China \\ ${ }^{2}$ Changjiang River Scientific Research Institute, Key Laboratory of Geotechnical Mechanics and Engineering of the Ministry of \\ Water Resources, Wuhan 430010, China
}

Correspondence should be addressed to Xu-dong Fu; xdfu@whu.edu.cn

Received 8 March 2016; Accepted 17 August 2016

Academic Editor: Hossein Moayedi

Copyright (c) 2016 Ben-jiao Zhang et al. This is an open access article distributed under the Creative Commons Attribution License, which permits unrestricted use, distribution, and reproduction in any medium, provided the original work is properly cited.

\begin{abstract}
The soft rock was simulated by cement, plaster, sand, water, and concrete hardening accelerator in this paper. Meanwhile, uniaxial compressive strength tests and triaxial compression tests were conducted to study the mechanical properties of simulated soft rock samples. Model tests on a single pile socketed in simulated soft rock under axial cyclic loading were conducted by using a device which combined test apparatus with a GCTS dynamic triaxial system. Test results show that the optimal mix ratio is cement : plaster : medium sand : water : concrete hardening accelerator $=4.5 \%: 5.0 \%: 84.71 \%: 4.75 \%: 1.04 \%$. The static load ratio (SLR), cyclic load ratio (CLR), and the number of cycles affect the accumulated deformation and cyclic secant modulus of the pile head. The accumulated deformation increases with increasing numbers of cycles. However, the cyclic secant modulus of pile head increases and then decreases with the growth in the number of cycles and finally remains stable after 50 cycles. According to the test results, the development of accumulated settlement was analysed. Finally, an empirical formula for accumulated settlement, considering the effects of the number of cycles, the static load ratio, the cyclic load ratio, and the uniaxial compressive strength, is proposed which can be used for feasibility studies or preliminary design of pile foundations on soft rock subjected to traffic loading.
\end{abstract}

\section{Introduction}

Socketed shafts are widely used in high-rise buildings, largespan bridges, large-scale aqueducts, and offshore oil drilling platforms and other projects, which have the advantages of high capacity, small settlement, stable pile bearing capacity, and good aseismic performance. Soft rock is broadly distributed: mudstone and shale account for about $50 \%$ of the earth's surface rocks, and rock-socketed pile foundations in soft rock have been widely used. In railway and highway construction, pile foundations would be the first choice because of their high capacity, low settlement, and so forth. Cyclic loading, such as that imposed by traffic, affects the accumulated settlement and bearing capacity of pile foundations. Cyclic loading is sparsely considered in design or covered by existing specifications. So it is of importance to study the effect of cyclic loading on the bearing capacity, stiffness, and accumulated settlement.
Many scholars have investigated the dynamic response of pile foundations subjected to cyclic axial loads [1-3]. Chan and Hanna [4] showed that the cyclic response of displacement piles in sand is affected by the number of cycles, their frequency, the mean shaft load, the shaft cyclic load amplitude, the pile depth, the loading history, and the properties of the surrounding sand. The development of settlement and axial force under cyclic loading were studied and the accumulated settlement and tip resistance increased with increasing numbers of cycles but the side friction gradually decreased. This was mainly caused by a decrease in horizontal stress under cyclic loading. Poulos [5] conducted small-scale model tests in sand and found that the critical cyclic loading amplitude causing large deformations was 0.70.8 under cyclic loading over 1000 cycles. Al-Douri and Poulos [6] studied the permanent accumulated displacement of a model pile under constant and variable amplitude and cyclic loading in calcareous sand. A numerical analysis was 
used to predict the cyclic behaviour of the model piles. Comparisons between the measured and predicted results showed reasonable agreement. Tsuha et al. [7] and Jardine and Standing [8] studied the settlement characteristics and shear stress, inter alia, of model pile under cyclic load in sand. Triaxial cyclic pile responses were categorised as stable, unstable, or metastable according to the number of cycles. Le Kouby et al. [9] and D'Aguiar et al. [10] found that the bearing capacity of a pile would be significantly improved when the amplitude of any cyclic loading was optimised. Bekki et al. [11] indicated that the side friction on a pile would show strain hardening because of the phenomenon of shear dilatancy under a large number of cycles. Chen et al. [12] and Ren [13] studied the cyclic performance of pile foundation subjected to long-term axial cyclic loading in silt.

Although many scholars have studied the accumulated settlement and bearing capacity characteristics of pile foundations under cyclic load, the soils in these tests were mostly soft clay, sand, or silt. The cyclic loading of pile foundations in soft rock is rarely reported since the excavation and transportation of natural soft rock might be costly. Synthetic material can be readily made and reproduced in identical batches in the laboratory. In addition, the simulated soft rock made by synthetic material has similar physical and mechanical properties to the natural soft rock. So the simulated soft rock is used widely to replace the natural soft rock in the laboratory model experiments. The selection of the best ratio of similar material has an important impact on the accuracy of model tests.

In view of the above, a test device, combining selfdesigned test apparatus with an American GCTS dynamic triaxial test-rig, was used to conduct cyclic loading tests on a single model pile socketed in simulated soft rock. This work studied the influence of static load ratio and cyclic load ratio on the accumulated settlement of the pile head and the cyclic secant modulus of pile head. This provided the experimental basis for the assessment of the response characteristics of a pile socketed in soft rock under dynamic cyclic loading.

\section{Mechanical Properties of Simulated Soft Rock}

The simulated soft rock was a mixture of cement, plaster, medium sand, water, and concrete hardening accelerator. To get the optimal mix proportions, various mixes were made and subjected to unconfined compressive strength testing on $\Phi 50 \mathrm{~mm} \times H 100 \mathrm{~mm}$ specimens. The optimal mix ratio was cement:plaster:medium sand: water: concrete hardening accelerator $=4.5 \%: 5.0 \%: 84.71 \%: 4.75 \%: 1.04 \%$. To acquire the mechanical parameters of uniaxial compressive strength values, deformation modulus, cohesion, and internal friction angle, uniaxial compressive strength tests and triaxial compression tests were carried out.

2.1. Uniaxial Compressive Strength Tests. The UCS tests were conducted on GCTS dynamic triaxial test equipment. The compressive strength and quasistatic Young's modulus $M$ were derived according to ASTM from uniaxial compressive

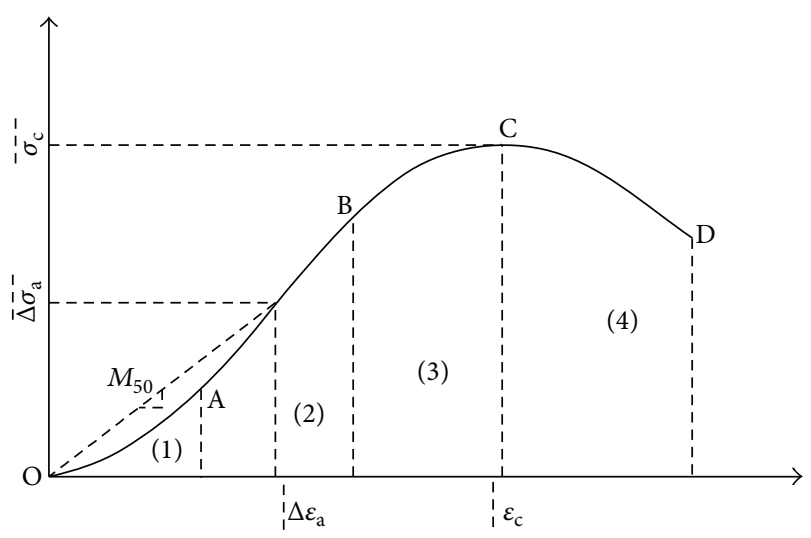

FIgURE 1: Typical compressive stress-strain curve of simulated soft rock.

tests at loading at a rate of $0.05 \mathrm{~mm} / \mathrm{min}$. Four samples were manufactured for each ratio and the average values were taken as final results of uniaxial compressive strength and deformation modulus. The unconfined compressive strength of the simulated soft rock sample is $1.44 \mathrm{MPa}$ and the deformation modulus is $157.88 \mathrm{MPa}$. The typical compressive stress-strain curve is shown in Figure 1.

As shown in Figure 1, the typical stress-strain curve of the simulated soft rock samples is made up of four stages: (1) the first stage OA is the compression phase, the inner pores and microcracks of samples are compressed, the stress-strain curve shows the convex type, and deformation modulus increases; (2) the second stage $\mathrm{AB}$ is an elastic phase, samples show elasticity, the stress-strain curve is a linear type, and the inside particles of samples are compressed but without breakage; meanwhile, deformation modulus stages are relatively stable; (3) the third stage $\mathrm{BC}$ is a plastic stage, the stress-strain curve is a nonlinear type, samples are gradually damaged until they reach to peak stress, and the deformation modulus decreases; (4) the fourth stage CD is a failure stage, stress-strain curve is sharply declining and it shows strain softening; the cracks are extending until the samples are completely damaged. The simulated soft rock samples are tension destruction under uniaxial compression; and the crack propagation direction is consistent with the loading direction. This characteristic is exactly similar to that of natural soft rock. So the method can commendably simulate the soft rock.

2.2. Triaxial Compression Tests. To research the characteristics of deviatoric stress-axial strain and volumetric strain relationship of simulated soft rock specimen under various confining pressures, obtaining its cohesion and internal friction angle, triaxial compression tests were conducted. The proportion of triaxial samples is as follows: cement:plaster:medium sand:water: concrete hardening accelerator $=4.5 \%: 5.0 \%: 84.71 \%: 4.75 \%: 1.04 \%$. And the specimen size is a diameter of $101 \mathrm{~mm}$ and a length of $200 \mathrm{~mm}$. The triaxial compression tests were conducted on GDS dynamic triaxial test equipment. The tests were controlled by strain at loading at the rate of $0.2 \mathrm{~mm} / \mathrm{min}$. 
TABLE 1: Triaxial compression tests and results.

\begin{tabular}{lccccccc}
\hline Sample number & $\begin{array}{c}\text { Confining } \\
\text { pressure } \\
(\mathrm{MPa})\end{array}$ & $\begin{array}{c}\text { Peak stress } \\
(\mathrm{MPa})\end{array}$ & $\begin{array}{c}\text { Failure strain } \\
(\%)\end{array}$ & $\begin{array}{c}\text { Maximum } \\
\text { volume } \\
\text { shrinkage } \\
(\%)\end{array}$ & $\begin{array}{c}\text { Shear } \\
\text { contraction } \\
\text { strain }(\%)\end{array}$ & $\begin{array}{c}\text { Initial } \\
\text { tangent } \\
\text { modulus } \\
(\mathrm{MPa})\end{array}$ & $\begin{array}{c}\text { Cohesion } \\
(\mathrm{MPa})\end{array}$ \\
\hline 1 & 0.01 & 1.51 & 1.132 & 0.331 & 0.807 & 162.16 & $\begin{array}{c}\text { Internal } \\
\text { friction angle } \\
\left({ }^{\circ}\right)\end{array}$ \\
2 & 0.10 & 2.12 & 1.547 & 0.416 & 0.881 & 174.05 & 0.344 \\
3 & 0.30 & 3.13 & 1.921 & 0.527 & 1.153 & 230.96 & 43.4 \\
4 & 0.50 & 3.61 & 2.195 & 0.717 & 1.424 & 243.36 & \\
\hline
\end{tabular}

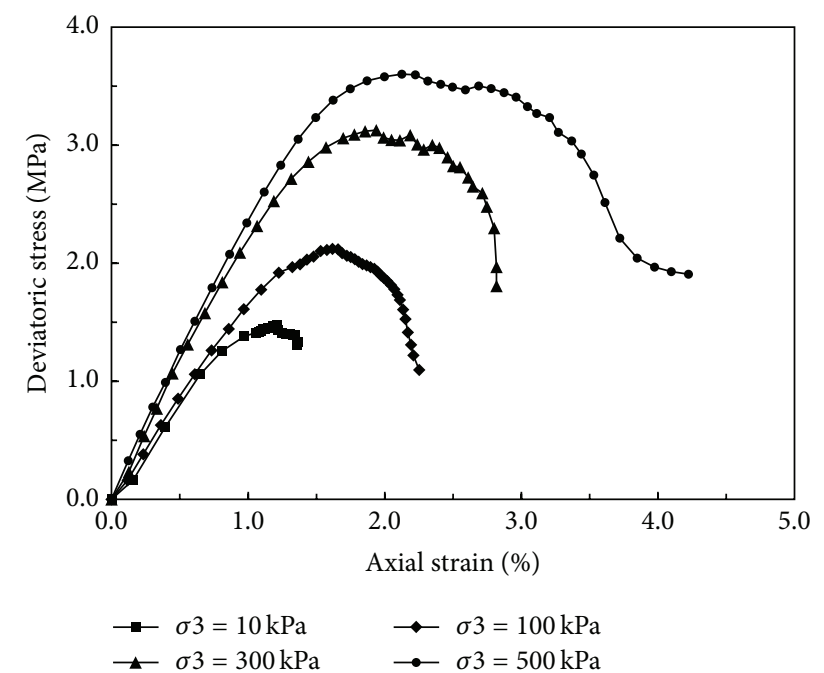

FIGURE 2: Deviatoric stress-axial strain curves of simulated soft rock specimen.

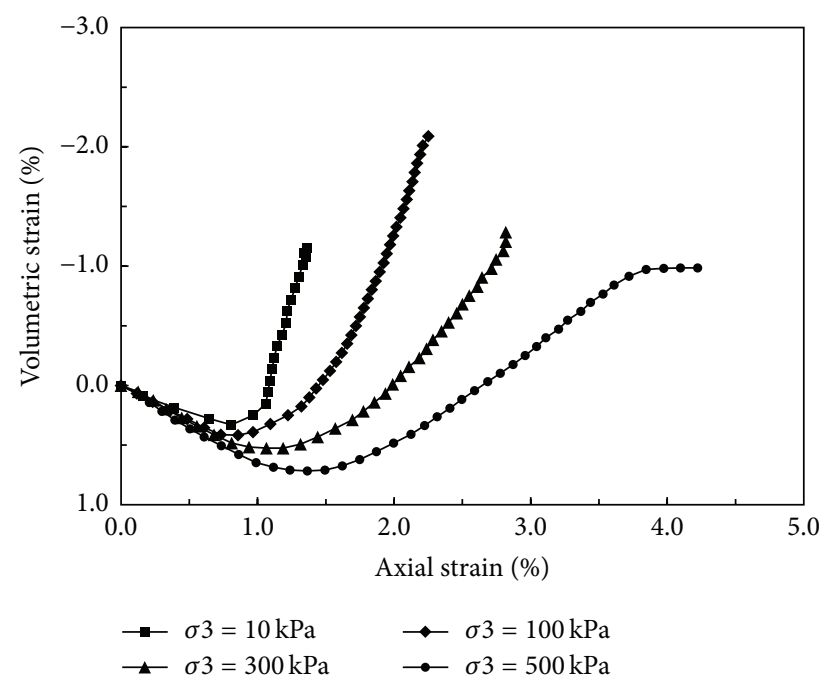

FIGURE 3: Volumetric strain-axial strain curves of simulated soft rock specimen.

The testing programme and results are shown in Table 1. Figure 2 shows the deviatoric stress-axial strain curves; Figure 3 displays the volumetric strain-axial strain curves. Figure 4 shows the relationship of confining pressures and deformation modulus and Figure 5 is Mohr's circles and strength envelope of simulated soft rock specimen.

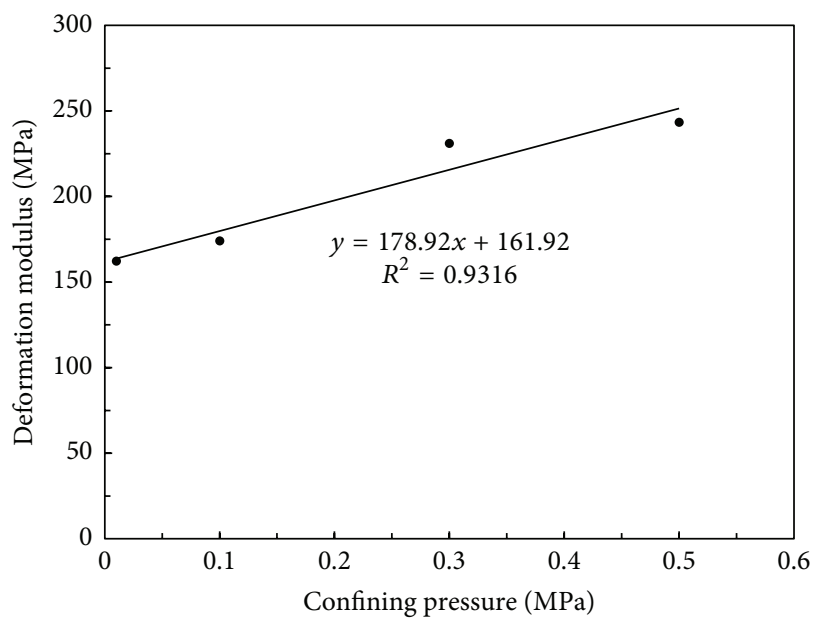

FIGURE 4: Relationship of confining pressures and deformation modulus of simulated soft rock specimen.

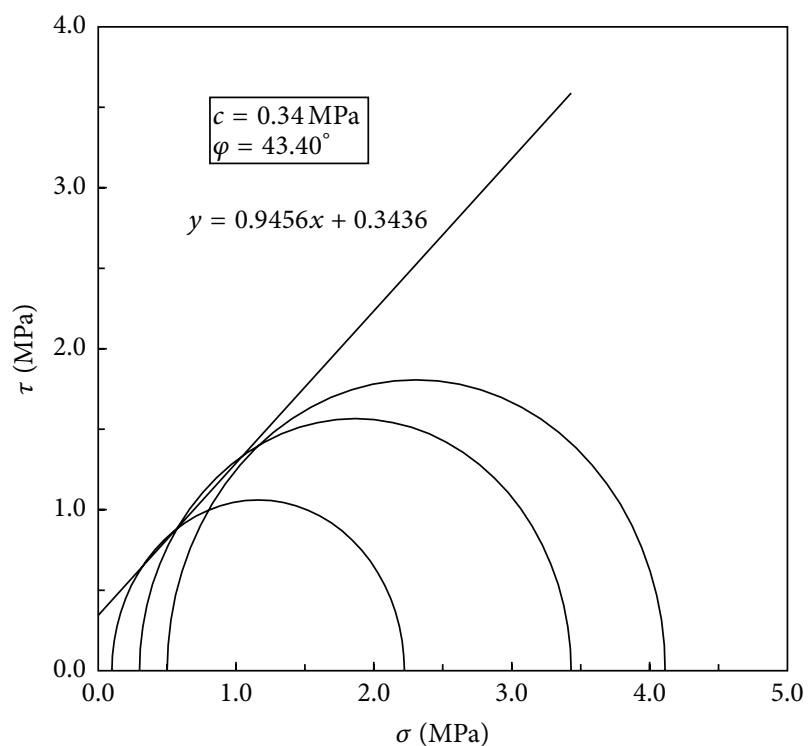

FIGURE 5: Mohr's circles and strength envelope of simulated soft rock specimen.

From Figures 2-5, it can be seen that the strength, the deformation modulus, and failure strain all increase with the growth of confining pressures. The strength is in a range of $1.51-3.61 \mathrm{MPa}$, the initial tangent modulus is in a range of $162.16-243.34 \mathrm{MPa}$, and the failure strain is between 
$1.13 \%$ and $2.19 \%$. The samples were applied to consolidation under confining pressures. Triaxial compression tests were conducted when the deformation and volume changes were stable. The inner pore was compacted with compression. So compared with uniaxial compressive strength tests, the compression phase is unconspicuous in triaxial compression tests. Complete stress-strain curves of simulated soft rock specimens under different confining pressures were constituted by three stages: the first stage is the elastic phase, and the stress-strain curve is a linear type; the second stage is the plastic stage, and the stress-strain curve is a nonlinear type; the third stage is the failure stage. The relationships of confining pressure and strength as well as the deformation modulus are basically linear types. And with the increase of confining pressure, the "stalemate" time of the failure strain is longer. It means a slower decline of descending stage. Under the compression of confining pressure and axial compressive force of the samples, shear contraction presents first, followed by the performance of dilatancy. And the strain of shear dilatancy is smaller than the strain of peak strength. It means that the samples present shear dilatancy firstly and then the strength reaches to the peak. The maximum shear contraction volumetric strain of the samples is $0.33 \%-0.72 \%$ and the shear contraction axial strain is $0.81 \%-1.43 \%$. The higher the confining pressure is, the larger the shear contraction strain is. It can be seen that the cohesion is about $0.34 \mathrm{MPa}$ and the internal friction angle is $43.40^{\circ}$ from Mohr's circles which are drawn by the results of triaxial tests and the confining pressure is $100 \mathrm{kPa}, 300 \mathrm{kPa}$, and $500 \mathrm{kPa}$, respectively.

According to the results of UCS tests and triaxial compression tests, mechanical parameters of uniaxial compressive strength values, modulus of deformation, cohesion, and internal friction angle are all in the range of that of natural soft rock. Meanwhile, the deviatoric stress-axial strain curves of simulated soft rock are exactly similar to that of natural soft rock. So the synthetic materials of cement, gypsum powder, medium sand, water, and concrete hardening accelerator can be used to simulate the natural soft rock.

\section{Experiment Design}

3.1. Test Device. Tests of a single model pile's bearing characteristics under axial cyclic loading were conducted on an American GCTS dynamic triaxial test-rig [14] (Figure 6).

The model pile was made of aluminium alloy bar (Type 2A12) with a diameter of $25 \mathrm{~mm}$ and a length of $500 \mathrm{~mm}$. The model pile is knurled on the surface of the pile tip over a $300 \mathrm{~mm}$ length and to a depth of $0.5 \mathrm{~mm}$. The knurling allowed good bonding with the simulated soft rock.

3.2. Sample Preparation. The model sample was stratified for preparation. To ensure homogeneity, the height of each layer of compacted soil was controlled according to its density. A certain amount of soft rock material substitute was placed into the bottom of the calibration chamber: the model pile was then installed vertically in the middle of the chamber and fixed with clamps. Finally the soft rock material was filled around the model pile and compacted with a hammer.

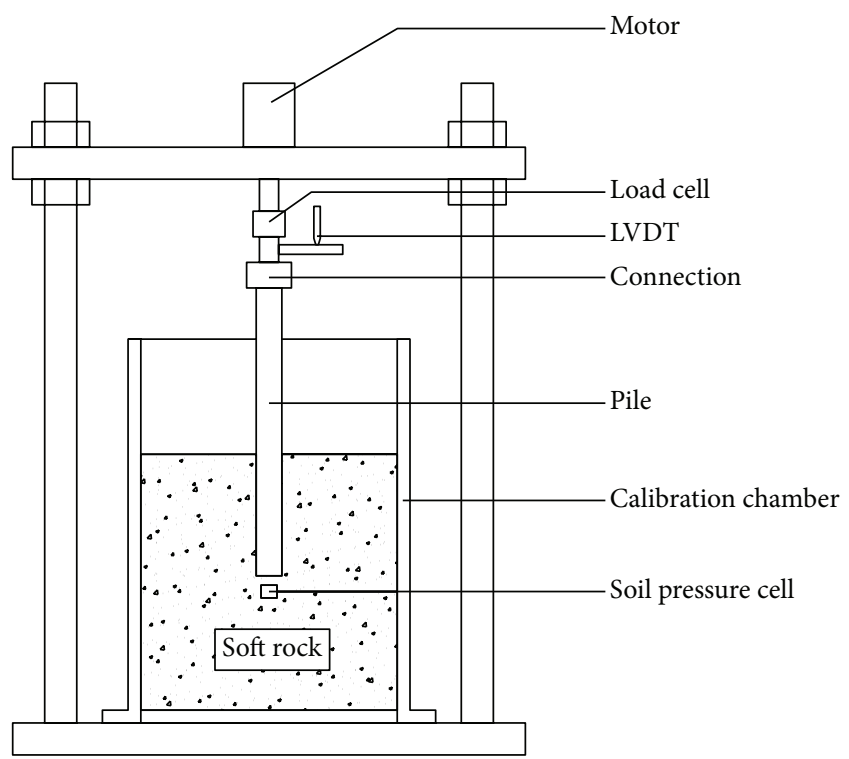

FIgURE 6: Test device.

The homogeneity of the whole specimen was important. The rock-socketed depth of the model piles was $5 d$ ( $d$ being the pile diameter).

After preparation, the model was covered with a rubber membrane for curing in constant humidity surroundings for seven days before testing.

3.3. Cyclic Loading Tests. The frequency of pile foundation cyclic loading tests ranges from 0.002 to $0.1 \mathrm{~Hz}$. According to other test results on bridge and railway pile foundations, most are subjected to dynamic load at 0.2 to $3.0 \mathrm{~Hz}$. The frequency used here was $0.2 \mathrm{~Hz}$ and the data were sampled at $50 \mathrm{~Hz}$. The effect of frequency on the response characteristics of pile will be analysed in future research. The sinusoidal waveform cyclic load, applied to the model pile head, was servo-controlled and defined as

$$
P(t)=P_{s}+\frac{1}{2} P_{c}+\frac{1}{2} P_{c} \sin (\omega t),
$$

where $P(t)$ represents the cyclic loading applied to the model pile head by the actuator; $P_{s}$ is the static load; $P_{c}$ is the amplitude of cyclic loading; $\omega$ is the circular frequency of cyclic loading, $\omega=2 \pi f$. The cyclic loading is as shown in Figure 7.

As shown in Figure 7, the static load ratio is defined as SLR $=P_{s} / P_{u}$; the cyclic load ratio is defined as CLR $=P_{c} / P_{u}$, and $P_{u}$ is the ultimate bearing capacity.

This work mainly focuses on the dynamic response characteristics of a single pile under one-way compression stress cyclic loading. It should satisfy the following relationship:

$$
0 \leq \mathrm{CLR}+\mathrm{SLR} \leq 1 .
$$

3.4. Test Programme. Static loading tests were carried out to ensure the vertical ultimate bearing capacity of single pile when the strength of simulated soft rock reached stabilisation. 


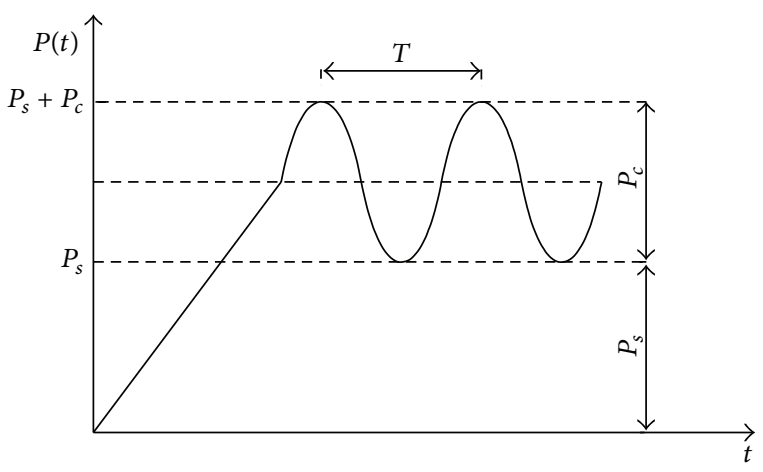

FIGURE 7: The cyclic loading regime.

TABLE 2: Cyclic loading test programme.

\begin{tabular}{lcccc}
\hline Test number & Pile diameter $(\mathrm{mm})$ & SLR & CLR & $\begin{array}{c}\text { Number of } \\
\text { cycles }\end{array}$ \\
\hline MP1 & 24 & 0.00 & 0.60 & 1000 \\
MP2 & 24 & 0.10 & 0.40 & 1000 \\
MP3 & 24 & 0.10 & 0.60 & 1000 \\
MP4 & 24 & 0.10 & 0.80 & 1000 \\
MP5 & 24 & 0.20 & 0.20 & 1000 \\
MP6 & 24 & 0.20 & 0.40 & 1000 \\
MP7 & 24 & 0.20 & 0.60 & 1000 \\
MP8 & 24 & 0.30 & 0.20 & 1000 \\
MP9 & 24 & 0.30 & 0.40 & 1000 \\
\hline
\end{tabular}

Then the cyclic loading tests were conducted under different SLRs and CLRs. After that, the static loading tests were carried out again to identify the influence of cyclic loading on the static bearing capacity. The SLR and CLR are considered here at a frequency of $0.2 \mathrm{~Hz}$. The testing programme is summarised in Table 2.

The static loading tests used quick maintenance loading, as recommended by ASTM [15]: the failure criterion of the pile foundation was a sharp increasing settlement in pile head. For dynamic loading tests, the tests were stopped when they met one of two criteria: (1) the number of cycles reaching 1000 or (2) the occurrence of a sharp increasing settlement in pile head.

\section{Analysis of Test Results}

4.1. Accumulated Settlement of Pile Head. Under vertical cyclic loading, the soft rock under the pile tip was subjected to cyclic compressive stress; meanwhile the pile-rock interface was subjected to repeated shear stress. Cyclic loading leads to soft rock particle rearrangement and a certain amount of residual plastic deformation. The accumulated settlement of the pile head was the macro-behavioural manifestation of that particulate rearrangement. Figure 8 shows the loaddisplacement curve of model pile $\mathrm{B}$ at $\mathrm{SLR}=0.30$ and CLR $=$ 0.40 . Figure 9 shows the accumulated displacement-number of cycles $(N)$ curves under different CLRs at SLR $=0.10$.

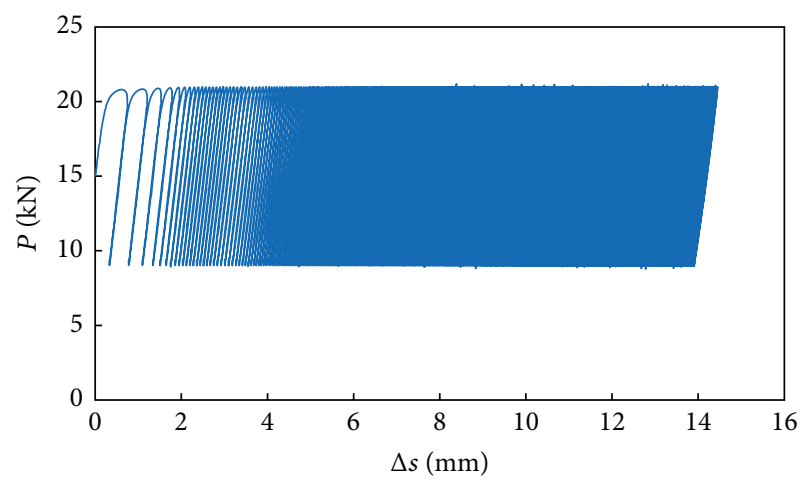

FIGURE 8: Cyclic load-displacement curve of model pile B (SLR = $0.30, \mathrm{CLR}=0.40$ ).

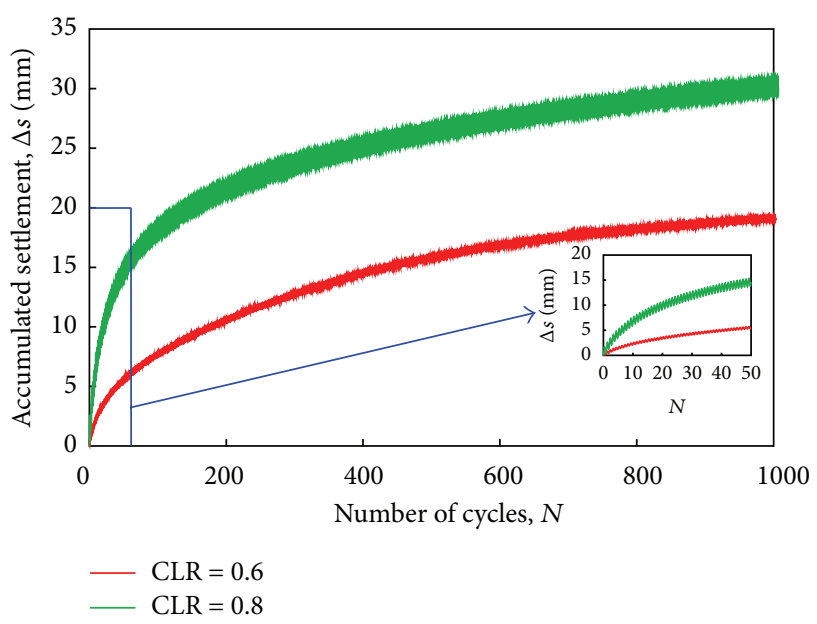

FIGURE 9: Development of accumulated displacement during dynamic loading tests $(S L R=0.10)$.

Figure 10 shows the accumulated displacement- $N$ curves under different SLRs at CLR $=0.60$.

From Figures 9 and 10, the accumulated displacement of the pile head was related to SLR, CLR, $N$, and the uniaxial compressive strength of the soft rock $\left(R_{f}\right)$. That is,

$$
\Delta s(N)=f\left(\operatorname{SLR}, \mathrm{CLR}, N, R_{f}\right) .
$$

The displacement decreases with increasing $R_{f}$. However, the displacement increases with increasing SLR, CLR, and $N$.

To understand better the increase in accumulated displacement, the accumulated settlement results were normalised. The nondimensional settlement of the pile was proposed, and it can be used to predict the accumulated settlement of the pile in situ. The nondimensional settlement of the pile head under $N$ load cycles can be expressed as

$$
\frac{\Delta s(N)}{s_{s}}=\frac{s_{N}-s_{0}}{s_{s}}
$$

where $s_{N}$ is the maximum settlement of the pile head under $N$ load cycles; $s_{0}$ is the deformation without cyclic loading; $s_{s}$ is the displacement at the same load, 


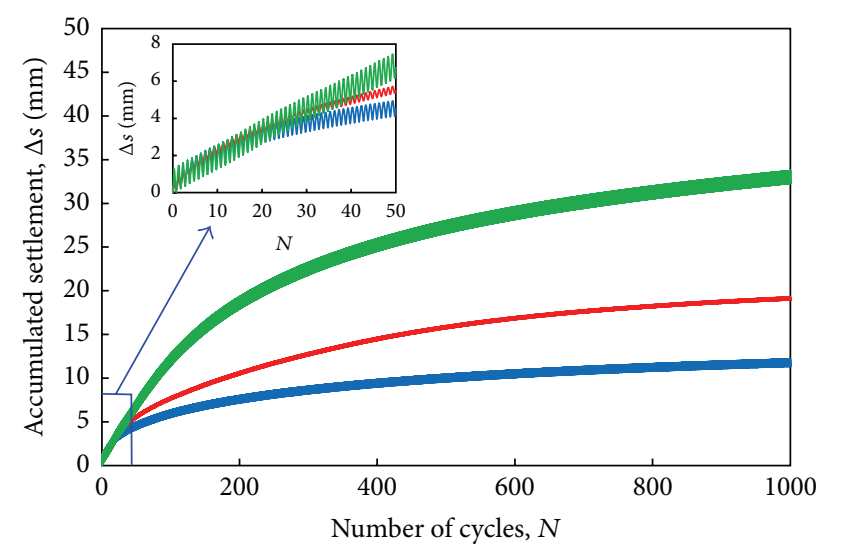

SLR $=0.0$

- SLR $=0.1$

- SLR $=0.2$

Figure 10: Development of accumulated displacement during dynamic loading tests $(C L R=0.60)$.

given by $\left((\mathrm{SLR}+\mathrm{CLR}) \times Q_{u}\right)$ under static load. Thence, the accumulated displacements are plotted in double logarithmic coordinates (Figure 11).

Figure 11 shows that the relationship between nondimensional accumulated settlement and number of cycles can be matched by a power function; that is, it shows a linear relationship in log-log coordinates. So the accumulated settlement under cyclic loading can be expressed as

$$
\frac{\Delta s(N)}{s_{s}}=\frac{1}{R_{f}^{k}} A N^{b}
$$

where $A$ and $b$ are best-fit parameters. The slopes of the accumulated settlement curves are similar, so $b$ is regarded as a constant. The effect of cyclic loading on parameter $A$ is given by

$$
A=a(\operatorname{SLR}+1)^{m}(\mathrm{CLR})^{n},
$$

where $a, m$, and $n$ are model parameters. When CLR $=0, A=$ 0 : this means that there is no cyclic accumulated settlement under static loading. When SLR $=0, A=a(\mathrm{CLR})^{n}$. The accumulated settlement is mainly determined by CLR.

From (5) and (6), it follows that

$$
\frac{\Delta s(N)}{s_{s}}=\frac{1}{R_{f}^{k}} a(\mathrm{SLR}+1)^{m}(\mathrm{CLR})^{n} N^{b},
$$

where $R_{f}$ is the uniaxial compressive strength $(1.44 \mathrm{MPa}$ here) and $k$ is the influence coefficient (taken as 1 here).

To obtain the value of the aforementioned model parameters, (7) was used to match the results in Figure 11. The model parameters obtained by back-analysis are summarised in Table 3.

Substituting model parameters $R_{f}$ and $k$ into (7), the final prediction of the accumulated settlement of the pile head under cyclic loading tests is

$$
\frac{\Delta s(N)}{s_{s}}=0.16(\mathrm{SLR}+1)^{4.09}(\mathrm{CLR})^{1.72} N^{0.254}
$$

TABLE 3: Back-analysis results: model parameters for accumulated displacement.

\begin{tabular}{lcccc}
\hline Parameter & $a$ & $b$ & $m$ & $N$ \\
\hline Value & 0.231 & 4.090 & 1.720 & 0.254 \\
\hline
\end{tabular}

As seen from Figure 11, the theoretical calculations and experimental results are consistent and the model can incorporate the effect of cyclic loading on accumulated settlement.

The proposed computational model can predict the tests results at $R_{f}=1.44 \mathrm{MPa}$ and $k=1$. The value of $k$ should be further verified if the model is to be extended to other strengths of soft rock.

4.2. Cyclic Secant Modulus. Figure 12 shows the loadsettlement hysteresis loops of a model pile. It can be seen that, with the generation of residual deformation, load-settlement hysteresis loops are not closed, while with increasing of numbers of cycles, residual deformation gradually stabilises and the hysteresis loops also tend to close [16]. A hysteresis loop includes four stages: loading phase $\mathrm{AB}$ with respect to the initial static compression state; unloading phase $\mathrm{BC}$ after peak load; unloading phase CD with respect to the initial static compression state; and loading phase DE after the minimum load. Residual deformation mainly occurs in phase AB. The secant modulus of the load-settlement curve should be calculated without using residual deformation data. Figure 13 shows the nonclosed load-settlement hysteresis loops and Figure 14 shows the variation of cyclic secant modulus under different CLRs.

As seen in Figures 12 and 13, the cyclic secant modulus of the settlement curve can be expressed by

$$
M_{s, c}=\frac{P_{c}}{\rho_{c}}\left(\frac{\mathrm{MN}}{\mathrm{m}}\right)
$$

where $P_{c}$ is the full amplitude of the cyclic load; $\rho_{c}$ is the amplitude of the dynamic settlement which is the difference between the settlement at point $\mathrm{A}$ and the settlement at point $B$ in a single cycle.

Figure 14 shows that the cyclic secant modulus $M_{s, c}$ firstly increases and then decreases and finally tends to a stable value as $N$ increases. The value of $M_{s, c}$ reaches its peak at $6 \leq N$ $\leq 8$ while $M_{s, c}$ reaches a stable value at $N \approx 50$ cycles. The soft rock particles at the pile tip were rearranged under this cyclic loading. The secant modulus increased rapidly because the soft rock was compressed and then the secant modulus began to decrease and eventually reached a constant value as the soft rock became damaged. The secant modulus decreases marginally and its stable value is about $85 \%$ to $95 \%$ of its maximum value. The final stable secant modulus is still higher (by about 30\%) than that of the initial stiffness. The cyclic secant modulus decreases with increasing CLR. The greater the CLR, the more damaged the soft rock particles. Here, the cyclic secant modulus was between 10.25 and $26.43 \mathrm{MN} / \mathrm{m}$. 


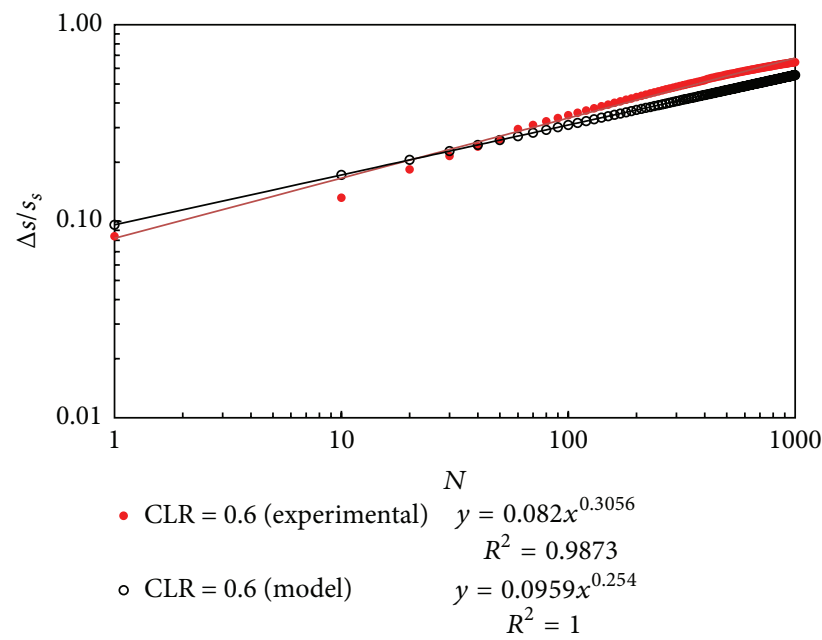

(a)

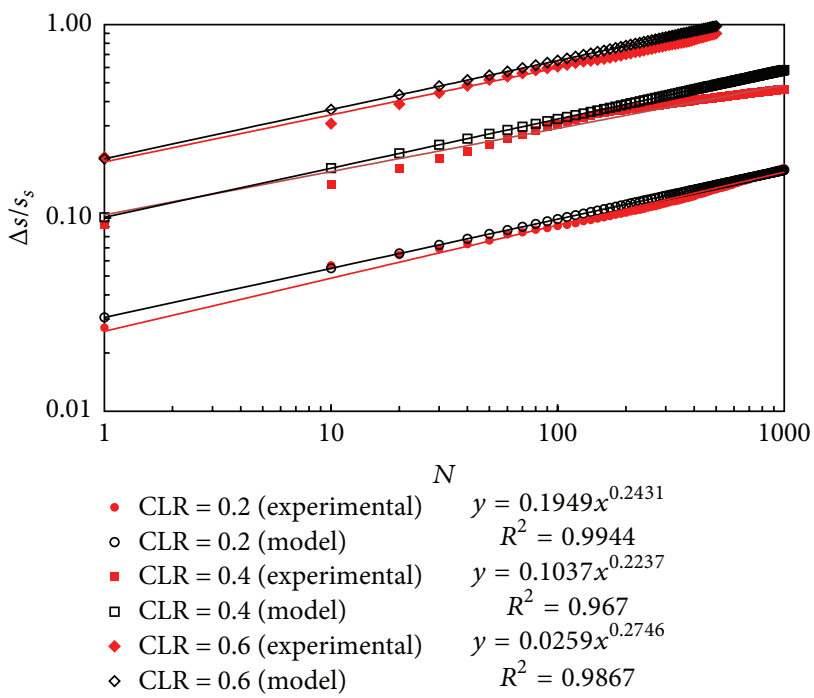

(c)

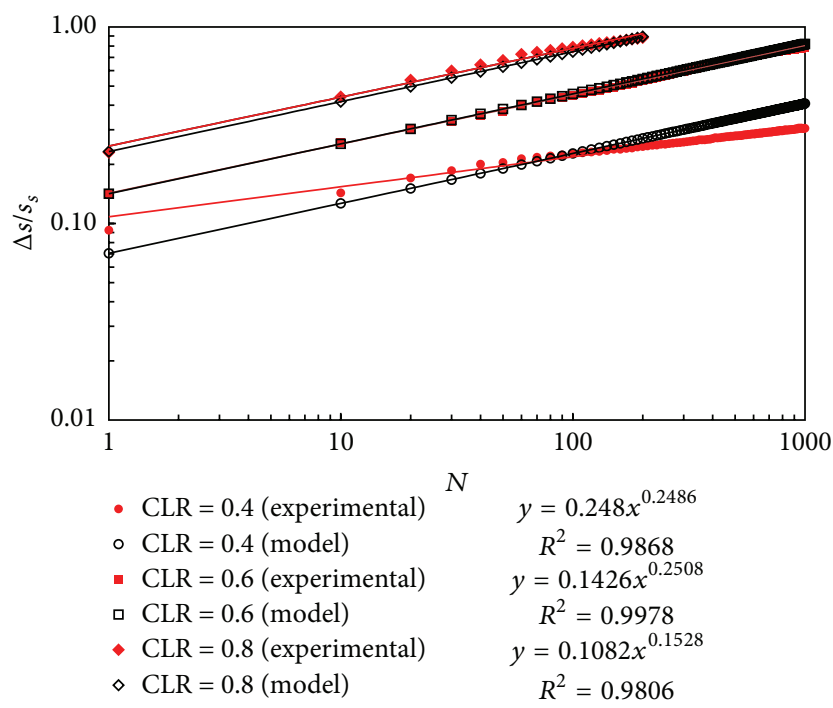

(b)

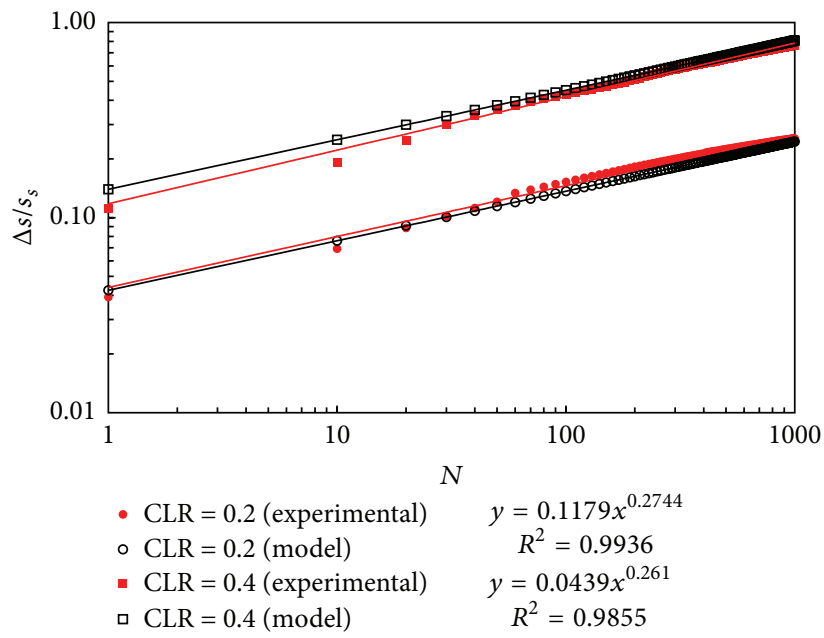

(d)

FIGURE 11: Variation of nondimensional accumulated displacement during dynamic load tests: (a) SLR = 0.0; (b) SLR = 0.1; (c) SLR = 0.2; (d) $\operatorname{SLR}=0.3$.

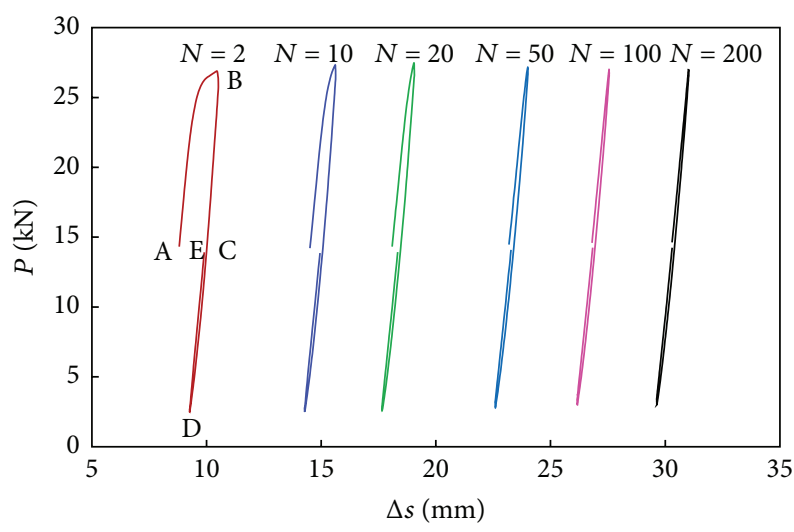

FIGURE 12: Load-settlement hysteresis loops for various values of $N$.

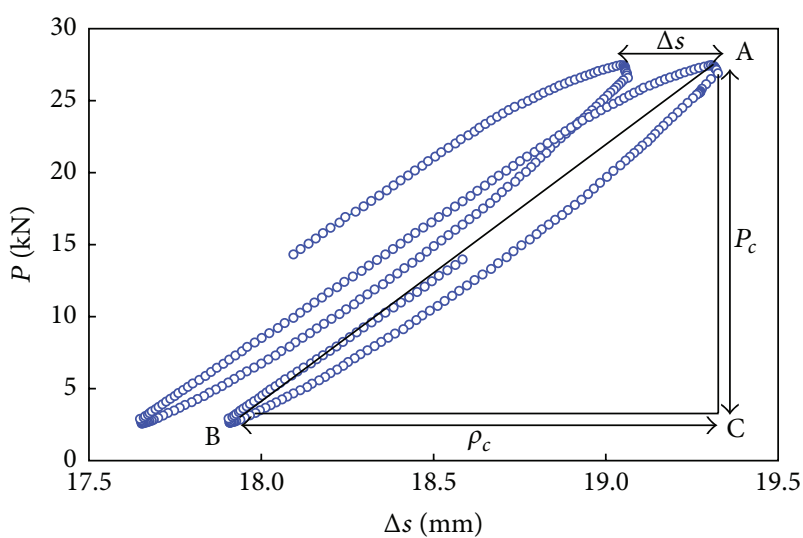

FIGURE 13: Nonclosed load-settlement hysteresis loops. 


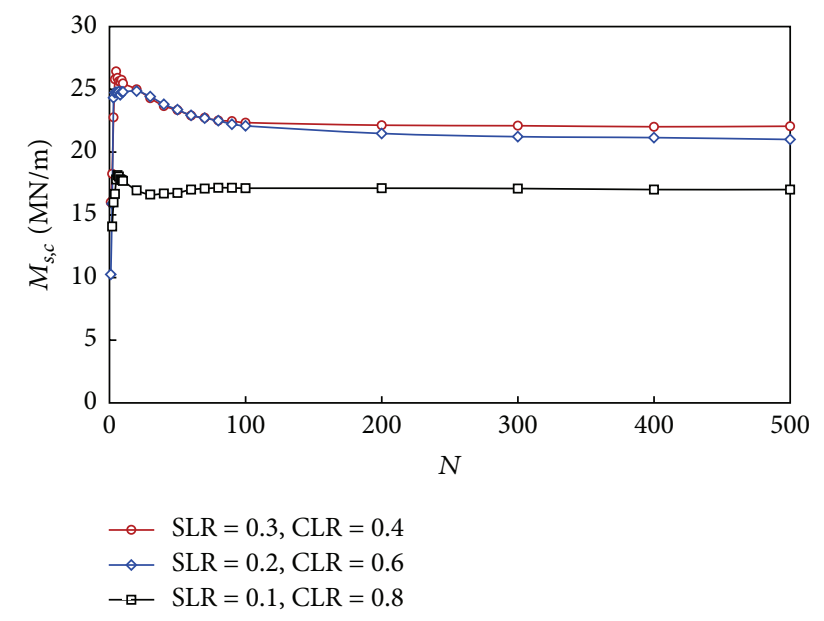

FIgURE 14: Variation of cyclic secant modulus for different CLRs.

\section{Conclusions}

Through the analysis of the response of a single pile socketed in soft rock under cyclic loading, the following conclusions can be obtained:

(1) According to the results of uniaxial compressive strength tests, the optimal mix ratio is cement: plaster:medium sand: water:concrete hardening accelerator $=4.5 \%: 5.0 \%: 84.71 \%: 4.75 \%: 1.04 \%$. The unconfined compressive strength of the simulated soft rock sample is $1.44 \mathrm{MPa}$ and the deformation modulus is $157.88 \mathrm{MPa}$.

(2) The strength, the deformation modulus, and failure strain all increase with the growth of confining pressures. The strength is in a range of 1.51-3.61 MPa, the initial tangent modulus is in a range of $162.16-243.34 \mathrm{MPa}$, and the failure strain is between $1.13 \%$ and $2.19 \%$.

(3) CLR and SLR affect the accumulated settlement. The accumulated settlement increases with increasing CLR and SLR. However, the ratio of accumulated settlement at each loading cycle decreases gradually with the increasing number of loading cycles. The relationship between nondimensional accumulated settlement of the pile head and the number of cycles can be represented by a power function; that is, it shows a linear relationship when plotted in log-log coordinates. A computational model of the accumulated settlement of the pile head is proposed which can be used for feasibility studies or the preliminary design, of pile foundations on soft rock subjected to traffic or other cyclic loading.

(4) The cyclic secant modulus firstly increases and then decreases and finally tends to a stable value with increasing numbers of cycles. The modulus reaches a peak at $6 \leq N \leq 8$ while the modulus reaches a stable value at $N \approx 50$. Meanwhile, the stable value is about $85 \%$ to $95 \%$ of its maximum value. The final stable secant modulus is still higher (by about 30\%) than its initial value.

\section{Competing Interests}

The authors declare that there are no competing interests regarding the publication of this paper.

\section{Acknowledgments}

The authors acknowledge the support from the National Natural Science Foundation of China (Grant nos. 51378403 and 51309028). The assistance given to the authors at various times by Mr. Zi-feng Qiu and Mr. Bu Lv is much appreciated.

\section{References}

[1] C. Y. Lee, "Cyclic response of axially loaded pile groups," Journal of Geotechnical Engineering, vol. 119, no. 9, pp. 1399-1413, 1993.

[2] J.-C. Dupla and J. Canou, "Cyclic pressuremeter loading and liquefaction properties of sands," Soils and Foundations, vol. 43, no. 2, pp. 17-31, 2003.

[3] Z. Li, M. D. Bolton, and S. K. Haigh, "Cyclic axial behaviour of piles and pile groups in sand," Canadian Geotechnical Journal, vol. 49, no. 9, pp. 1074-1087, 2012.

[4] S.-F. Chan and T. H. Hanna, "Repeated loading on single piles in sand," Journal of the Geotechnical Engineering Division, vol. 106, no. 2, pp. 171-188, 1980.

[5] H. G. Poulos, "Cyclic axial response of single pile," Journal of Geotechnical and Geoenvironmental Engineering, vol. 107, no. 1, pp. 41-58, 1981.

[6] R. H. Al-Douri and H. G. Poulos, "Predicted and observed cyclic performance of piles in calcareous sand," Journal of Geotechnical Engineering, vol. 121, no. 1, pp. 1-16, 1995.

[7] C. H. C. Tsuha, P. Y. Foray, R. J. Jardine, Z. X. Yang, M. Silva, and S. Rimoy, "Behaviour of displacement piles in sand under cyclic axial loading," Soils and Foundations, vol. 52, no. 3, pp. 393-410, 2012.

[8] R. J. Jardine and J. R. Standing, "Field axial cyclic loading experiments on piles driven in sand," Soils and Foundations, vol. 52, no. 4, pp. 723-736, 2012.

[9] A. Le Kouby, J. Canou, and J. C. Dupla, "Behaviour of model piles subjected to cyclic axial loading," in Proceedings of the Cyclic Behaviour of Soils and Liquefaction Phenomena, pp. 159166, Bochum, Germany, April 2004.

[10] S. C. D’Aguiar, F. Lopez-Caballero, A. Modaressi-FarahmandRazavi, and J. A. Santos, "Piles under cyclic loading: study of the friction fatigue and its importance in piles behavior," in Proceedings of the 17th International Conference on Soil Mechanics and Geotechnical Engineering (ICSMGE '09), vol. 2, pp. 1313-1316, Alexandria, Egypt, October 2009.

[11] H. Bekki, J. Canou, B. Tali, J.-C. Dupla, and A. Bouafia, "Evolution of local friction along a model pile shaft in a calibration chamber for a large number of loading cycles," Comptes Rendus - Mecanique, vol. 341, no. 6, pp. 499-507, 2013.

[12] R.-P. Chen, Y. Ren, B. Zhu, and Y.-M. Chen, "Deformation behaviour of single pile in silt under long-term cyclic axial loading," in Proceedings of the 18th International Conference on Soil Mechanics and Geotechnical Engineering, Paris, France, 2013. 
[13] Y. Ren, Model test and theoretical study on deformation behaviour of single piles to long-term cyclic axial loading [Ph.D. thesis], Zhejiang University, Hangzhou, China, 2013.

[14] X.-D. Fu, B. Huang, B.-J. Zhang, and C. Mei, "A physical model testing apparatus of bearing mechanism of socketed pile in weak rock by CT scanning," Patent ZL 201410213046.1, 2014.

[15] A.S.T.M. D1143-81, "Standard test method for piles under static axial compressive load," ASTM, 1994.

[16] B. Huang, X.-D. Fu, B.-J. Zhang, and Z.-F. Qiu, “Test technology and normalized characteristics of dynamic elastic modulus and damping ratio," Chinese Journal of Geotechnical Engineering, vol. 37, no. 4, pp. 659-666, 2015. 

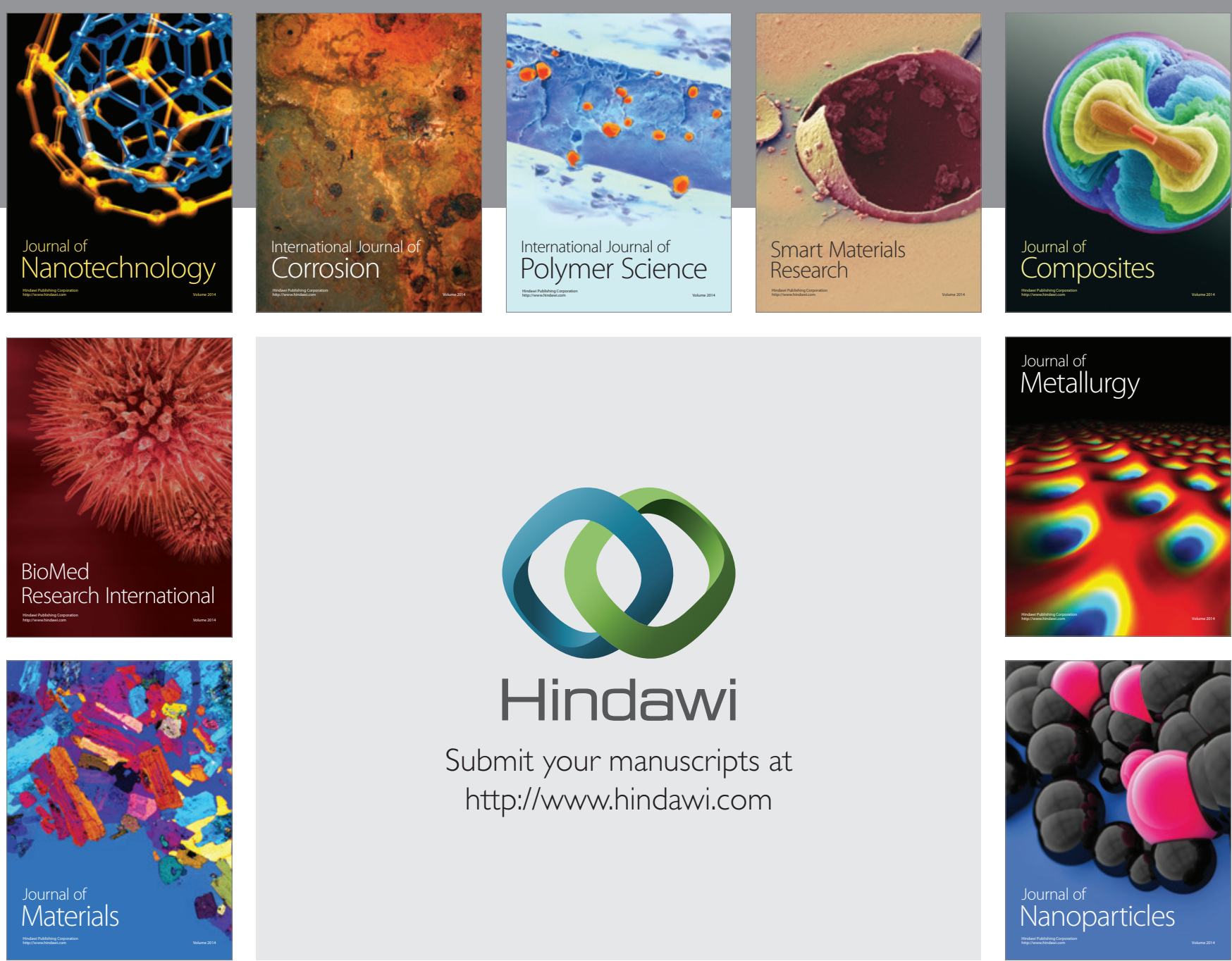

\section{Hindawi}

Submit your manuscripts at

http://www.hindawi.com

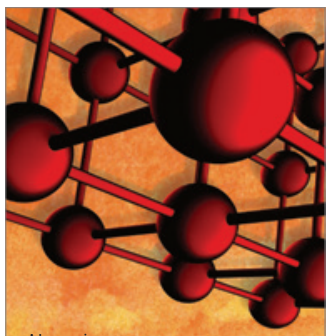

Materials Science and Engineering
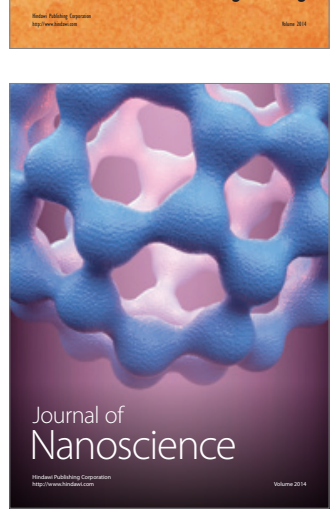
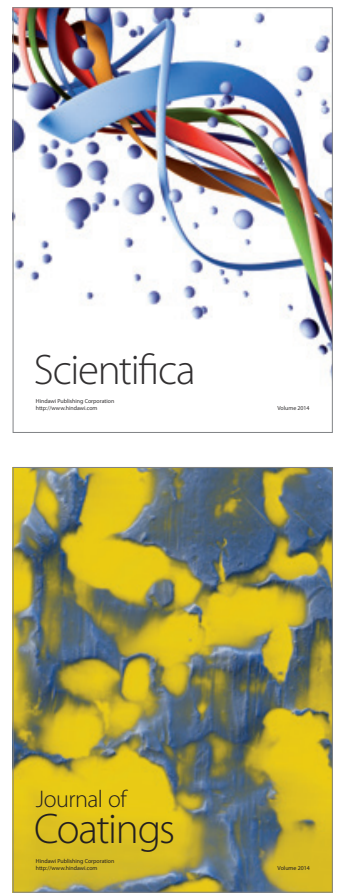
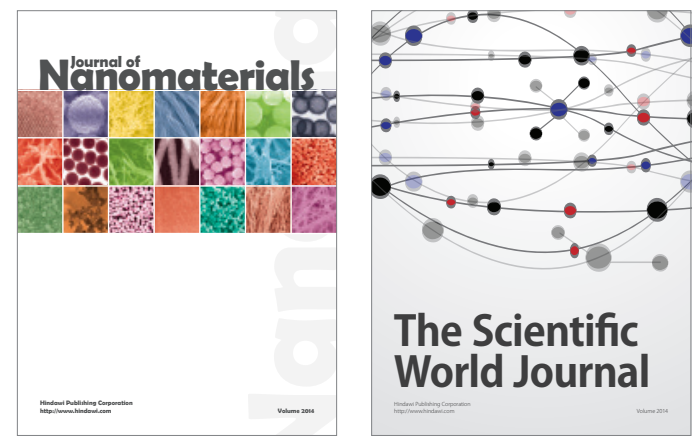

The Scientific World Journal
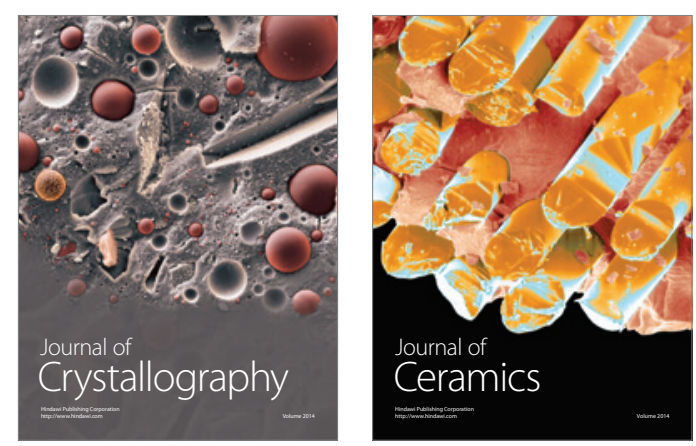
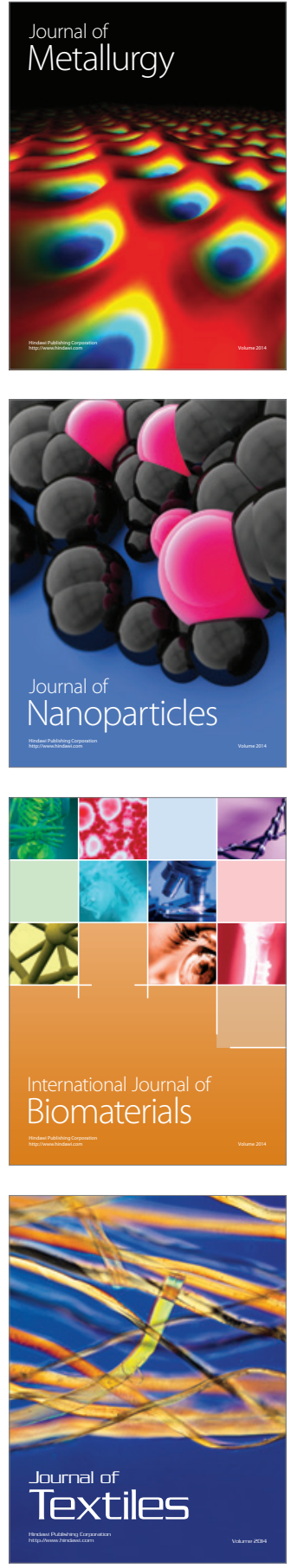